\title{
Diriger une PME. Introduction
}

Managing a SME. Introduction

\section{Thomas Amossé}

\section{(2) OpenEdition}

Journals

Édition électronique

URL : https://journals.openedition.org/travailemploi/5651

DOI : 10.4000/travailemploi.5651

ISSN : 1775-416X

Éditeur

DARES - Ministère du Travail

\section{Édition imprimée}

Date de publication : 15 juin 2012

Pagination : 5-8

ISSN : 0224-4365

\section{Référence électronique}

Thomas Amossé, « Diriger une PME. Introduction », Travail et Emploi [En ligne], 130 | avril-juin 2012,

mis en ligne le 01 janvier 2013, consulté le 30 juillet 2021. URL : http://journals.openedition.org/ travailemploi/5651 ; DOI : https://doi.org/10.4000/travailemploi.5651 


\title{
Diriger une PME Introduction
}

\author{
Thomas Amossé (*)
}

Depuis sa création en 1979, la revue Travail et emploi a régulièrement publié des articles traitant des petites ou moyennes entreprises (PME). Selon les époques, les thèmes d'intérêt ont changé, qui témoignent des dispositifs que les pouvoirs publics ont spécifiquement consacrés à ces entreprises depuis trente ans : dans les années 1980, à la suite de la loi Auroux, les PME étaient décrites sous l'angle des lois sociales et modalités d'expression collective; la décennie suivante, elles le furent sous celui de la formation (en écho à la loi de 1991), de la gestion prévisionnelle des emplois et des compétences, et du recours à certains contrats aidés tels les contrats de retour à l'emploi après la crise de 1993. Au tournant du millénaire, c'est au prisme du temps de travail et de sa réduction que les PME ont de nouveau été examinées. Dans cet ensemble d'articles, une contribution (1) fait date, qui présente la synthèse d'un colloque organisé en 1994 sous l'égide du ministère du Travail. Reprenant l'intervention introductive de Jean Saglio, l'article retrace l'évolution du regard que les sciences sociales ont porté sur ces entreprises. Il dresse ensuite un état des connaissances quant à leurs éventuelles spécificité et homogénéité en matière de création/destruction d'emplois, d'innovation productive mais aussi d'organisation du travail.

La publication, près de vingt ans plus tard, d'un numéro intitulé «Diriger une PME» invite à reconsidérer le bilan d'étape établi alors. Les questions posées en 1994 sont-elles toujours d'actualité ? Les réponses apportées valent-elles encore? De nouvelles questions et de nouvelles réponses ont-elles émergé? L'objectif du numéro n'est certes pas de produire un état des lieux de la recherche concernant les PME sur les questions du travail et de l'emploi. Et notre introduction n'en a pas davantage l'ambition. Toutefois, les cinq recherches empiriques originales que propose cette nouvelle livraison de la revue engagent d'un point de vue disciplinaire, sociologie, sciences politiques et sciences de gestion et ont été sélectionnées à partir d'un large appel à contribution. Elles fournissent l'occasion de s'arrêter sur la question des PME et sur le rôle qu'y occupent les dirigeants.

Les PME constituent un objet relativement récent pour les sciences sociales du travail et de l'emploi. L'entreprise a en effet longtemps été pensée sur le modèle des grandes organisations, que la supériorité supposée de la division scientifique du travail posait comme un horizon nécessaire et inéluctable du capitalisme. Qualifiées de «traditionnelles », souvent familiales, les PME étaient alors vues comme des entreprises devant se moderniser afin de se développer et croître (2). Ce n'est qu'avec la remise en cause du fordisme dans les années 1970, crise économique et critique politique aidant, que les PME sont réellement devenues un enjeu de recherche. Leur meilleure santé économique face à la crise persistante dans les années 1980 a achevé de convaincre qu'une révision des modèles d'analyse antérieurs était nécessaire. S'est ainsi imposée l'idée que les petites et moyennes entreprises n'étaient pas des " grandes entreprises en réduction » (3) et qu'elles constituaient un observatoire privilégié pour rendre compte de la variété des configurations productives.

(*) Centre d'études de l'emploi; thomas.amosse@cee-recherche.fr

(1) Cf. Bruno Baranger (1995), «L'emploi et les relations sociales dans les PME. Compte rendu du colloque du 11 octobre $1994 »$, Travail et emploi, $\mathrm{n}^{\circ} 63$, pp. 89-99.

(2) L'article de Philippe Madinier (1983), «La persistance des très petites entreprises dans les activités non agricoles», Travail et emploi, $\mathrm{n}^{\circ} 16$, pp. 67-81, témoigne de la force de cette conception des PME. L'auteur y indique par exemple que «jusqu'à la fin des années soixante, l'unanimité se fait en France pour penser qu'une forte concentration est à la fois nécessaire et souhaitable dans tous les secteurs de l'activité économique» (p. 67).

(3) $C f$. Marie-Françoise Raveyre et Jean Saglio (1984), «Les systèmes industriels localisés : éléments pour une analyse sociologique des ensembles de PME industriels », Sociologie du travail, n², pp. 157-176. 
D'un point de vue théorique, l'émergence des PME comme objet de recherche a notamment accompagné le développement de l'économie des conventions ${ }^{(4)}$, et les débats en économie et en histoire(5) sur la performance des «districts industriels» (désignés parfois aussi comme des clusters), groupes de PME spécialisées ancrées dans un territoire. Au plan international, elle a soutenu la «multiple model theory» (6), qui reconnaît l'efficacité des diverses formes d'organisation du travail et questionne la complémentarité des facettes de l'activité des entreprises ${ }^{(7)}$. Dans un capitalisme mondialisé où les capacités d'adaptation sont particulièrement attendues des entreprises pour faire face à la concurrence et aux incertitudes existantes sur le marché des biens, du travail et des capitaux, les PME semblent pouvoir inspirer de nouveaux modèles. La «spécialisation souple » ${ }^{(8)}$ et l'organisation en flux tendus (ou lean production ${ }^{(9)}$ ) ont par exemple tenté de combiner les avantages de la petite série avec la production de masse, quand le concept d' «entreprise apprenante» ${ }^{(10)}$ paraît particulièrement adapté aux entreprises de taille limitée.

En parallèle et dans le prolongement de ce mouvement, le développement de la recherche en sciences de gestion a également favorisé dans les années 1990 les travaux portant sur les PME et l'entrepreunariat ${ }^{(11)}$, avec une attention particulière portée au rôle du dirigeant dans la stratégie et l'organisation du travail(12) et non plus seulement à sa personnalité ou à son profil social. Dans les années 2000, la dynamique ne s'est pas démentie, même si la complexification des liens financiers et commerciaux entre filiales et entités d'un groupe ou d'un réseau de franchise, entre sous-traitants et donneurs d'ordre, rend certainement moins opérantes les analyses distinguant les entreprises selon un seul critère de taille. En économie, après une période d'intense concentration sur l'analyse des districts industriels et autres formes de spécialisation flexible territorialisée, des travaux récents ont par exemple porté sur le lien entre la structure du capital ou le type de gouvernance de l'entreprise (institutionnel versus familial) et les pratiques managériales. L'opposition entre les grandes, moyennes et petites entreprises est ainsi moins tranchée qu'auparavant. La taille est toujours un élément clé de compréhension de la diversité des entreprises, mais elle doit être complétée par des éléments relatifs aux formes d'organisation du travail, au rôle du dirigeant, à la structure capitalistique, etc.

Quoi qu'il en soit, en quarante ans, l'image des PME a radicalement changé. Elles ne sont plus ces unités archaïques amenées à disparaître avec le progrès économique. Elles sont désormais considérées comme des éléments essentiels d'un capitalisme fait d'une pluralité de types d'entreprise qui s'inscrivent dans des réseaux complexes de relations financières et commerciales. Aujourd'hui symboles de souplesse et de réactivité, de croissance potentielle et de gisement d'emplois localisés quand les grandes entreprises mondialisées restructurent leurs unités de production en Europe, elles sont l'objet de toutes les attentions de la part des pouvoirs publics qui en font une clé du développement économique, que ce soit en France ou au niveau européen ( $c f$. le Small Business Act de 2008). Il est vrai, plusieurs facteurs structurels, qu'il serait difficile de lister de façon exhaustive, ont pu contribuer à renforcer les avantages relatifs de la petite taille : la révolution numérique et les progrès de l'automatisation peuvent permettre le développement de certaines activités sans main-d'œuvre

(4) $C f$. la parution en 1989 du numéro spécial «L'économie des conventions» dans la Revue économique (vol. 40, n²) sous la direction de François Eymard-Duvernay ou en 1993, le livre de Robert Salais et Michael Storper, Les mondes de production : enquête sur l'identité économique de la France, Paris, Éditions de l'École des hautes études en sciences sociales.

(5) Cf. par exemple Giacomo Becattini (1992), «Le district industriel : milieu créatif», Espaces et sociétés, n 66-67, pp. 147-163, Claude Courlet et Bernard Pecqueur (1992), «Les systèmes industriels localisés en France : un nouveau modèle de développement», in Georges Benko et Alain Lipietz (dir.), Les Régions qui gagnent. Districts et réseaux : les nouveaux paradigmes de la géographie économique, Paris, PUF, pp. 81-102, ou plus récemment Florent Le Bot, Cédric Perrin (dir.) (2011), Les chemins de l'industrialisation en Espagne et en France. Les PME et le développement des territoires (XVIIIe-XXIe siècles), Bruxelles, Peter Lang.

(6) Cf. Eileen Appelbaum et Rosemary Batt (1994), The new American workplace: transforming work systems in the United States, London, ILR Press.

(7) Cf. Paul Milgrom et John Roberts (1990), «The economics of modern manufacturing: technology, strategy and organization», The American Economic Review, vol. 80, n 3, pp. 511-528.

(8) Cf. Michael Piore et Charles Sabel (1989), Les chemins de la prospérité : de la production de masse à la spécialisation souple, Paris, Hachette.

(9) Cf. James Womack, Daniel Jones et Daniel Ross (1993), The machine that changed the world, New York, Rawson Associates.

(10) Cf. Nonaka Ikojiro et Hirotaka Takeuchi (1997), La connaissance créatrice, la dynamique de l'entreprise apprenante, Bruxelles, De Boeck Université.

(11) En témoigne par exemple la création de deux associations francophones en la matière (l'AIREPME et l'Académie de l'entrepreunariat) et de nombreux diplômes universitaires sur ces thématiques.

(12) Cf. William Gartner (1988), "Who is an entrepreneur?», American Journal of Small Business, vol. 12, n4, pp. 11-32 qui a contribué à renouveler l'approche suivie jusqu'alors. 
abondante (dans les services informatiques comme l'industrie); la demande de services localisés aux personnes dans le secteur sanitaire et social peut se traduire par la création d'établissements de soin de taille moyenne; la concurrence des pays à bas coût de la main-d'œuvre peut obliger à repenser le positionnement stratégique de certaines entreprises, et les inviter à miser sur des courtes séries sur des marchés de niche plutôt que sur de la production de masse.

Les handicaps de la petite taille demeurent toutefois, faits d'économies d'échelle et de ressources de financement limitées, d'une organisation du travail moins formalisée et plus souvent exposée à l'urgence. La petite taille peut aussi être le signe d'un déficit de maturité ou de réflexion stratégique sur le développement de l'entreprise, d'un apprentissage encore à faire, pour le dirigeant comme le collectif de travail. Elle peut enfin être le lieu de relations interpersonnelles fortes, parfois familiales, entre le dirigeant et son personnel qui, en cas de conflit, peut se transformer en management autoritaire et offre moins de médiation de recours pour les salariés en l'absence de syndicat ou de direction des ressources humaines (DRH). Les cinq articles qui composent ce numéro ne montrent de fait aucune supériorité des PME, bien loin d'être devenues un nouveau one best way entrepreneurial. De même qu'en matière d'emplois, les PME sont à la fois les plus dynamiques et les plus exposées au risque de faillite, les situations décrites dans les textes qui suivent mettent en évidence des situations contrastées, où la taille, moyenne ou petite des entreprises, apparaît tour à tour comme un avantage ou un inconvénient. Et où le dirigeant peut tout autant sembler subir que maîtriser les événements.

Dans son article, Caroline Mazaud décrit une mutation gestionnaire de l'artisanat. Suivant un assouplissement du cadre réglementaire, les entreprises artisanales seraient ainsi, de plus en plus, des entreprises comme les autres : l'auteure y observe un moindre exercice du «métier» artisanal par des dirigeants qui se sont formés à la gestion et à la prospection commerciale, ou sont issus des classes moyennes ou supérieures par le biais de reconversions en cours de carrière. Cette transformation, qui peut être considérée comme une modernisation du secteur, n'est pas sans risque du point de vue de la maîtrise des savoir-faire et de l'équilibre social de la filière artisanale où les débouchés de carrière des ouvriers qualifiés pourraient se trouver à l'avenir limités.

Baptiste Giraud s'intéresse quant à lui à une facette moins classique de l'activité des dirigeants de PME : leur participation à une organisation professionnelle locale. Étudiant un $c l u b$ d'entreprise dans un bassin d'emploi rural, il montre que l'engagement dans cette structure constitue une composante à part entière de l'activité des chefs des plus petites entreprises : leur participation ne s'accompagne pas seulement d'une recherche de notabilité; elle permet de nouer des relations commerciales, de bénéficier d'expériences passées d'autres chefs d'entreprise comme parfois de conseils ou d'appuis juridiques. Mais cette composante de l'activité est inégalement partagée, puisque les directeurs de filiales de groupes (inter) nationaux (après absorption d'anciennes entreprises familiales) se tiennent plus souvent en retrait du club. Baptiste Giraud montre ainsi comment les transformations du capitalisme ont modifié à la fois la structuration patronale locale et la manière dont ces entreprises sont dirigées, leur inscription se faisant désormais davantage au sein du groupe que du territoire.

Dans sa contribution, Caroline Debray décrit un phénomène relativement peu connu en sociologie et économie du travail et de l'emploi : les «hypogroupes», c'est-à-dire des groupes composés de petites entreprises. À partir de quatre études de cas suivis sur une période de douze ans, elle pose la question de la constitution et du développement de ces groupes comme alternative à la croissance des petites entreprises. Phénomène en plein essor, bien que difficile à mesurer d'un point de vue statistique, ce type nouveau de PME est une manière autre que directement organisationnelle de préserver les bénéfices de la petite taille tout en poursuivant le développement de son entreprise. Les questions de la professionnalisation du management et de la formalisation du contrôle s'y trouvent plus particulièrement analysées.

À côté de cette innovation «capitalistique», le texte de Brigitte Charles-Pauvers et Nathalie Schieb-Bienfait décrit un chantier d'innovation organisationnelle à partir de l'étude d'une entreprise, SCOP BTB, qui présente des similitudes avec les entreprises apprenantes décrites dans la littérature. Constituée en société coopérative de production, cette entreprise associe le dirigeant et les salariés actionnaires de façon originale dans un projet d'entreprise où de nombreux espaces de régulation et structures de concertation existent : commissions et autres groupes de travail sont créés de façon souple, sur des bases hiérarchiques, fonctionnelles ou thématiques; la participation, l'expérimentation et l'initiative sont encouragées. Il peut s'agir par exemple de la création d'une commission à 
l'initiative d'un ou plusieurs salariés visant à travailler sur de nouveaux matériaux ou à développer des savoir-faire. Si la taille de l'entreprise (environ 125 salariés) n'est a priori pas ici un critère nécessaire ni suffisant, il est évident que la souplesse permise par un effectif limité en même temps que les économies d'échelle et la diversité des métiers garanties par un nombre suffisant de salariés font de cette organisation un exemple potentiellement spécifique de la moyenne entreprise.

Dans leur article, Isabelle Tilloy-Alphonse, Antoine Masingue et Jean-Michel Pottier décrivent la mise en place dans quatre PME du Valenciennois d'un dispositif de politique publique, la Préparation opérationnelle pour l'emploi (POE), qui facilite le recrutement par le financement de la formation d'un demandeur d'emploi. A priori adapté aux PME, un tel dispositif n'est pourtant pas sans y poser un certain nombre de difficultés, ce qui souligne l'importance des freins à la mise en œuvre de formations professionnelles dans ces entreprises. Contrastant avec les innovations mises en évidence dans les deux textes précédents, cette contribution rappelle l'éloignement des dirigeants de PME de l'univers des politiques de l'emploi et de la formation professionnelle, suggérant qu'il ne pourrait être résolu que par l'intervention d'un «traducteur».

Ces articles montrent tout à la fois que dans l'univers des PME coexistent les traits classiques d'entreprises où la petite taille est un handicap relatif et d'autres où elle est transformée en atout, pour développer des formes originales d'organisation par exemple. Ils témoignent en même temps des transformations récentes du capitalisme, avec un développement des fonctions gestionnaires et commerciales aussi dans les petites entreprises, et avec des logiques de groupes qui se développent et reconfigurent la manière de diriger. À la lecture du dossier, plusieurs chantiers de recherche restent encore ouverts pour mieux comprendre les pratiques professionnelles des dirigeants de PME et leurs liens d'intrication avec les grandes entreprises. Du côté de leurs pratiques gestionnaires, il faudrait mieux documenter par quelles voies les méthodes de management circulent et pénètrent les PME : mobilités externes de personnes (cadres notamment)? Reconfiguration de périmètre des grandes entreprises (filialisation, externalisation, acquisition)? Intervention de tiers extérieurs (consultants privés ou chargés de mission de dispositifs publics)? Du côté des relations externes, on cerne encore mal les relations des dirigeants de PME avec leurs clients donneurs d'ordres et surtout avec leurs banquiers, alors que ces relations de forte dépendance sont centrales pour des structures fragiles et plus sujettes que les grandes entreprises au risque de faillite. Ces questions, comme les transformations soulignées par le dossier, sont en partie communes aux grandes et moyennes, voire petites entreprises. Leur imbrication croissante, du fait de liens financiers et commerciaux toujours plus nombreux, invite plus largement à considérer que la diversité des entreprises ne peut, encore moins qu'auparavant, se résumer par une opposition selon la taille. 